\title{
miR-379/411 cluster regulates IL-18 and contributes to drug resistance in malignant pleural mesothelioma
}

\author{
KAZUO YAMAMOTO, MASAHIRO SEIKE, SUSUMU TAKEUCHI, CHIE SOENO, AKIHIKO MIYANAGA, \\ RINTARO NORO, YUJI MINEGISHI, KAORU KUBOTA and AKIHIKO GEMMA \\ Department of Pulmonary Medicine and Oncology, Graduate School of Medicine, \\ Nippon Medical School, Tokyo 113-8603, Japan
}

Received May 17, 2014; Accepted August 20, 2014

DOI: $10.3892 /$ or.2014.3481

\begin{abstract}
Malignant pleural mesothelioma (MPM) is a rapidly fatal malignancy that is increasing in incidence in Japan. In this study, we performed gene and microRNA (miRNA) expression profiling to identify novel therapeutic targets in MPM cells. Based on relative sensitivities to pemetrexed (PEM) and the histone deacetylase (HDAC) inhibitor, vorinostat (SAHA), $211 \mathrm{H}$ cells were determined to be the only sensitive MPM cell line out of the 6 tested. On the same series of cell lines, we performed whole genome transcriptomic profiling via DNA microarrays and pathway analysis of the derived data. Of particular note, IL-18 gene expression levels were significantly higher in the cell lines that were either drug resistant or displayed intermediate sensitivity, compared to the sensitive $211 \mathrm{H}$ cell line. Pathway analysis revealed IL-18 as an important gene associated with drug sensitivity of MPM cells. A relationship between IL-18 overexpression and drug resistance was also observed following targeted assessment of 10 cytokine genes using quantitative RT-PCR. miRNA expression profiles were evaluated in the MPM cell line panel in order to discern the mechanism of IL-18 induction in the drug-resistant lines. We found that miR-379 and miR-411 belonged to the same cluster of miRNAs located on chromosome $14 \mathrm{q} 32$ that commonly target the $I L-18$ gene. Luciferase reporter assays revealed that miR-379 and miR-411 directly target the $I L-18$ gene. Introduction of miR-379 plus miR-411, as well as IL-18 silencing, significantly suppressed the invasive capacity of MESO1 cells in vitro. Furthermore, the use of either PEM or SAHA together with miR-379 plus miR-411 mimics mediated increased sensitivity to these drugs in MESO1 cells. These results suggest that the miR-379/411 cluster may provide new therapeutic opportunities for advanced MPM patients, depending on the nature of $I L-18$ gene expression.
\end{abstract}

Correspondence to: Dr Masahiro Seike, Department of Pulmonary Medicine and Oncology, Graduate School of Medicine, Nippon Medical School, 1-1-5 Sendagi, Bunkyo-ku, Tokyo 113-8603, Japan E-mail: mseike@nms.ac.jp

Key words: IL-18, miR-379, miR-411, mesothelioma, histone deacetylase inhibitor

\section{Introduction}

Malignant pleural mesothelioma (MPM) is an aggressive tumor that arises from serosal cells and affected patients exhibit poor prognosis (1). The incidence of the disease is expected to steadily increase due to occupational asbestos exposure and is becoming recognized as a particular societal problem in Japan (2). Indeed, over 100,000 MPM-related deaths are predicted to occur within the next 40 years in Japan (3). Surgical resection is only possible in a minority of patients. In addition, the 5-year survival rate of patients that undergo surgical resection is less than $15 \%$ (4). Chemotherapy can be used as a standard therapy for MPM patients with advanced disease. In this context, a variety of drugs including cisplatin (CDDP), gemcitabine, and pemetrexed (PEM) have been used in different combinations. Chemotherapy with PEM and CDDP has yielded the best effect in MPM patients to date, with a response rate of $41 \%$ and a median survival time of 12 months (5). However, major concerns related to this treatment revolve around the short duration of response and rapid relapse. Therefore, new therapeutic modalities that have clinically beneficial effects are urgently required for appropriate management of MPM patients. Recently, the histone deacetylase (HDAC) inhibitor, vorinostat (SAHA), significantly enhanced CDDP-induced apoptosis in MPM cells (6). In addition, the HDAC inhibitor, valproate, in combination with CDDP and PEM, provided additional efficacy in respect to treatment of MPM cell line-derived xenografts in vivo (7). A phase I clinical trial of carboplatin and paclitaxel with SAHA including non-small cell lung cancer (NSCLC) and MPM showed significantly improved response rates and a trend towards enhanced survival (8). Therefore, combining platinum drugs with SAHA might be a promising therapeutic strategy in MPM.

Tumors employ several mechanisms to avoid or actively suppress anticancer immune responses (9). The secretion by tumor cells of soluble factors such as IL-6, IL-18 and TGF- $\beta$ can directly block T-cell proliferation, promote T-cell apoptosis, or confer resistance to tumor cells against T-cell attack (10). The inflammatory status of noncancerous lung tissue surrounding a tumor may also play an important role in promoting tumor progression and metastasis in lung adenocarcinoma (11). Among the tumor-specific cytokines, IL-18 plays a pivotal role 
in inflammation and the immune response (12-14). Moreover, IL-18 can also promote human cancer progression and metastasis (15). Understanding of the inflammatory status of human cancer may provide new insights in respect to diagnostic and therapeutic strategies.

MicroRNAs (miRNAs) are single-stranded 18-24 nt non-coding molecules that post-transcriptionally modulate gene expression through binding to 3'-UTRs of target mRNAs (16). miRNAs, which usually induce gene silencing, can function as either tumor suppressors or oncogenes (17). Recent studies have identified several miRNAs with diagnostic, prognostic and therapeutic potential in pulmonary malignant diseases including NSCLC and MPM (18-21). For example, the identification of low levels of miR-29c in MPM has provided both a new prognostic tool and has pointed to aberrant chromatin methylation as being involved in progression of the disease (20). A recent study demonstrated that loss of miR-145 affects tumorigenic properties of MPM cell lines (21). These findings have prompted the study of miRNA expression levels as potentially important diagnostic and prognostic tools in MPM.

In the present study, we analyzed the correlation between gene expression and antitumor effects of PEM and SAHA in MPM cell lines by DNA microarray-based whole transcriptome profiling and targeted RT-PCR analysis for 10 cytokine genes. We focused on IL-18 for further analysis among the identified genes associated with drug resistance in MPM cells. Furthermore, miRNAs within the miR-379/411 cluster contributed to drug resistance through the regulation of their target IL-18. Our research has revealed, for the first time, that IL-18 and the miR-379/411 cluster may have dual functions in tumor invasion and drug resistance in MPM.

\section{Materials and methods}

Cell culture. We used six MPM cell lines in the present study, as follows: NCI-H28, NCI-H2452 and ACC-MESO4 (epithelial-type), NCI-H2052 (sarcomatoid-type), ACC-MESO1 (fibroblast-type) and MSTO-211H (biphasic-type). NCI-H28, NCI-H2452, NCI-H2052 and MSTO-211H were purchased from the American Type Culture Collection (Manassas, VA, USA). ACC-MESO1 and ACC-MESO4 were obtained from the Riken Cell Bank (Tsukuba, Japan). MPM cells were maintained in RPMI-1640 (Gibco, Carlsbad, CA, USA) with 10\% fetal bovine serum (FBS; Gemini Bioproducts). These cell lines were obtained from 2008 to 2009, were amplified and frozen, and one aliquot of each was thawed for this project. All cells were routinely screened for the absence of mycoplasma. These cell lines were maintained in RPMI-1640 medium (Gibco) supplemented with 10\% FBS.

Drugs and growth-inhibition assay. PEM was purchased from Toronto Research Chemicals (North York, Canada). SAHA was purchased from Cayman Chemicals (Ann Arbor, MI, USA). Growth inhibition was assessed by the MTS assay to examine the effect of PEM and SAHA on the MPM cell lines as previously described (22). Cell suspensions $(5,000$ cells/well) were seeded into 96 -well plates and increasing concentrations of PEM and SAHA $(0,0.001,0.01$. $0.1,1.0,10$ and $100 \mu \mathrm{M})$ were added. After incubation at $37^{\circ} \mathrm{C}$ for $72 \mathrm{~h}$, MTS was added to each well and incubated at $37^{\circ} \mathrm{C}$ for $2 \mathrm{~h}$, after which the absorbance was measured with a test wavelength of $490 \mathrm{~nm}$ using a microplate reader (Dynatech MR7000; Dynatech, Billinghurst, UK). The $\mathrm{IC}_{50}$ value was defined as the concentration of PEM and SAHA needed for $50 \%$ reduction of growth and was calculated by SigmaPlot12 (Hulinks, Inc., Tokyo, Japan). Each experiment was performed independently three times. The corrected absorbance of each sample was calculated and compared with that of the untreated control.

RNA isolation and real-time quantitative reverse transcription-PCR. Total RNA was isolated from MPM cell lines, as previously described (11). The expression profiles of 10 cytokine genes [i.e. interleukin 1A (IL-1A), interleukin 2 (IL-2), interleukin 4 (IL-4), interleukin 6 (IL-6), interleukin 8 (IL-8), interleukin 10 (IL-10), interleukin 12A (IL-12A), interleukin 18 (IL-18), interleukin 24 (IL-24) and interleukin 27 (IL-27)] were examined by quantitative real-time PCR (qRT-PCR) analysis using specifically designed TaqMan Human Gene Expression Assays (Applied Biosystems, Foster City, CA, USA). GAPDH served as an endogenous control. Gene expression data (mean \pm SD from triplicate samples) are shown as $\Delta C$ t. The qRT-PCR assessment of gene expression was performed using the ABI Prism 7700 Sequence Detector system (Perkin Elmer/Applied Biosystems).

GeneChip and miRNA array analysis. High-density oligonucleotide array analysis was carried out using Affymetrix HG-U133A GeneChips (22,282 probe sets), as previously described (23). In terms of miRNA profiling, $5 \mu \mathrm{g}$ of total RNA was employed for hybridization on miRNA microarray chips containing 667 probes with the TaqMan ${ }^{\circledR}$ Array Human MicroRNA A+B Card Set ver. 2.0 (Life Technologies, Carlsbad, CA, USA) on a 7900 Real-Time PCR system (Applied Biosystems, Foster City, CA, USA), as previously described (24).

Oligonucleotidetransfection.Smallinterfering RNAs(siRNAs) targeting IL-18 were purchased from Dharmacon Research Inc. (Lafayette, CO, USA), and the homologous negative controls were obtained from Invitrogen. miR-379 and miR-411 mimics, and the control-mimic, were obtained from SigmaAldrich (St. Louis, MO, USA). IL-8 siRNAs and miR-379/411 mimics were transfected using Lipofectamine 2000 reagent $24 \mathrm{~h}$ after seeding, according to the manufacturer's instructions (Life Technologies). Transfections of siRNA and miRNA mimic complexes were added to cells at a final concentration of $50 \mathrm{nM}$.

Luciferase assay. Luciferase reporter constructs containing portions of the IL-18 3' UTRs were generated by GeneCopoeia, Inc. (Rockville, MD, USA). H28 cells were cultured in 24-well plates for $24 \mathrm{~h}$ and cotransfected with $100 \mathrm{ng} / \mu \mathrm{l}$ of IL-18 3' UTR reporter constructs and $50 \mathrm{~nm}$ of miR-379 mimic, miR-411 mimic, or control-mimic using Lipofectamine 2000 for 24 to $48 \mathrm{~h}$. After transfection, cells were harvested, lysed and assayed with a Dual-Luciferase Reporter assay kit (Promega, Madison, WI, USA) according to the manufacturer's instructions. Firefly luciferase activity 
Table I. $\mathrm{IC}_{50}$ values in 6 MPM cell lines responding to treatments with PEM and SAHA as determined by the MTS assay.

\begin{tabular}{lcccccc}
\hline & \multicolumn{5}{c}{$\begin{array}{c}\text { Cell line } \\
\text { (pathological type) }\end{array}$} \\
\cline { 2 - 7 } Treatment & $\begin{array}{c}211 \mathrm{H} \\
\text { (biphasic) }\end{array}$ & $\begin{array}{c}\mathrm{H} 28 \\
\text { (sarcoma) }\end{array}$ & $\begin{array}{c}\mathrm{H} 2052 \\
\text { (epithelial) }\end{array}$ & $\begin{array}{c}\text { H2452 } \\
\text { (epithelial) }\end{array}$ & $\begin{array}{c}\text { MESO1 } \\
\text { (fibroblast) }\end{array}$ & $\begin{array}{c}\text { MESO4 } \\
\text { (epithelial) }\end{array}$ \\
\hline PEM IC $_{50}(\mu \mathrm{M})$ & 0.07 & 0.07 & 0.57 & $>100$ & $>100$ & $>100$ \\
SAHA IC $_{50}(\mu \mathrm{M})$ & 3.3 & 9.1 & 7.2 & 7.2 & 8.1 & 9.2 \\
Drug sensitivity & Sensitive & Intermediate & Intermediate & Resistant & Resistant & Resistant \\
\hline
\end{tabular}

A

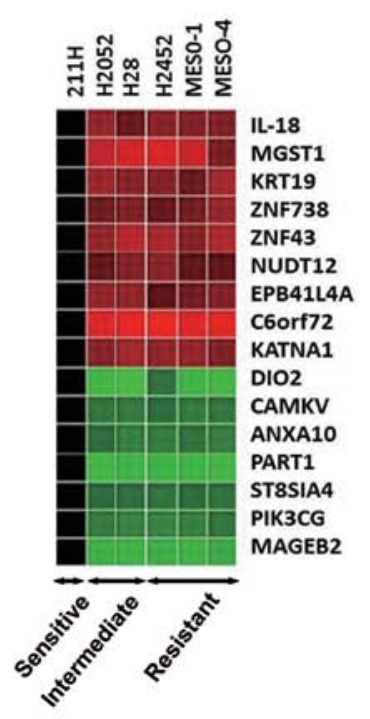

B

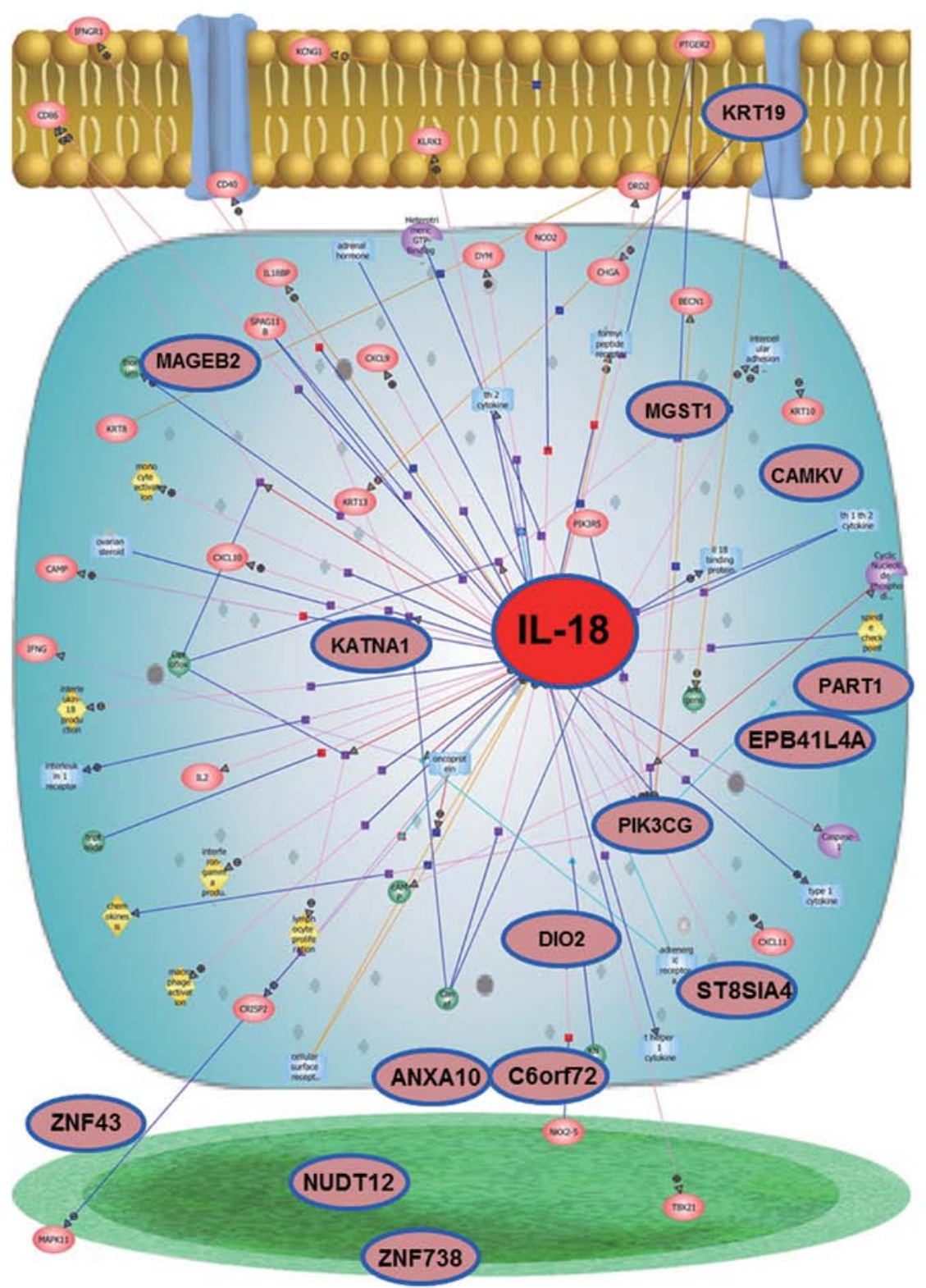

Figure 1. Genes that are differentially expressed between drug-sensitive and -resistant MPM cell lines. (A) Sixteen gene transcripts were differentially expressed between the drug-sensitive $211 \mathrm{H}$ cell line and the resistant/intermediate sensitive cell lines. Fold change $>1.5$; P-value $<0.05$. (B) Sixteen genes (blue circle) associated with drug responses were established by pathway analyses.

was normalized to Renilla luciferase activity for each transfected well. Each experiment was performed in duplicate and repeated three times.
Invasion assay. The ability of cells that either overexpressed miR-379 or miR-411, or were silenced for IL-18 to invade through a Transwell filter was measured using a Cytoselect 
A

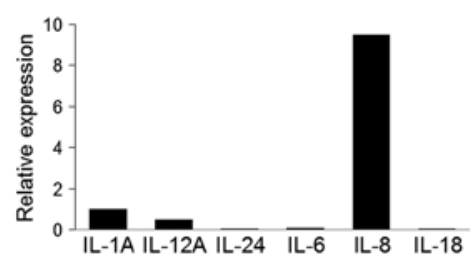

B

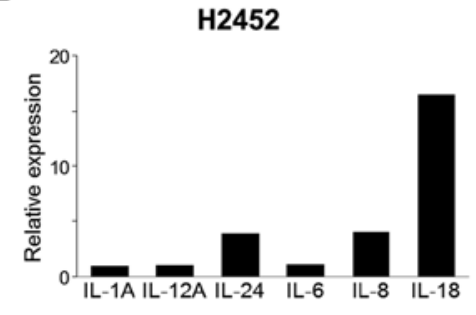

C

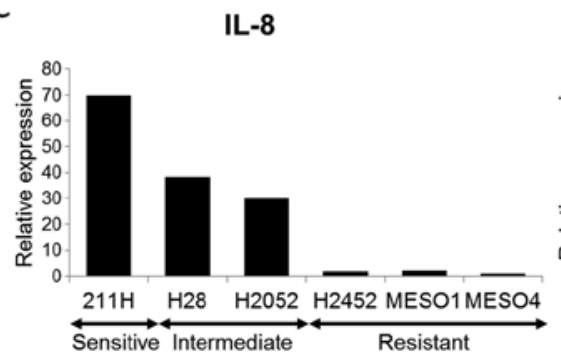

H28

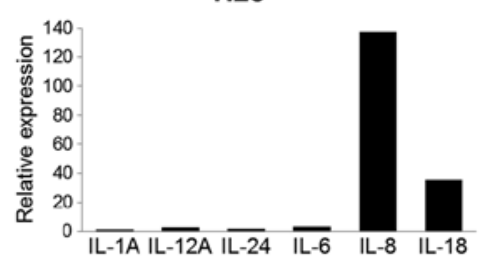

MESO1

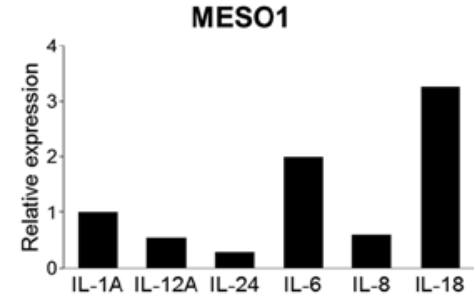

IL-18
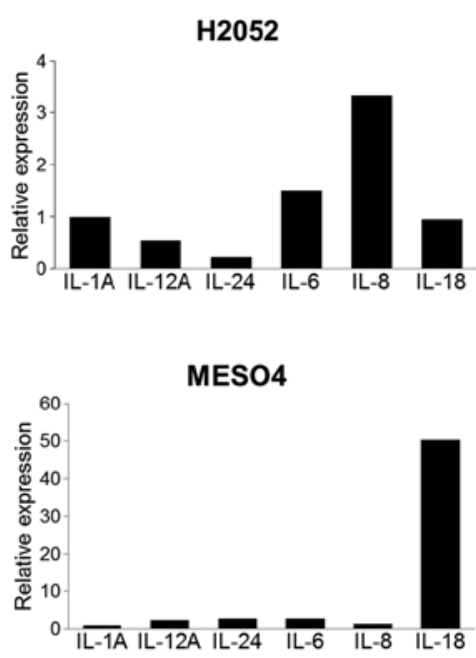

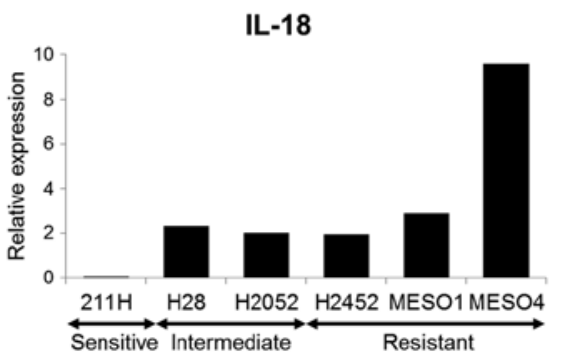

Figure 2. Cytokine gene expression profiling in MPM cell lines. (A) Expression profiles of 6 cytokine genes were assessed in the drug-sensitive and intermediate-sensitive cell lines by qRT-PCR analysis. Relative ratios of gene expression were calculated by comparison with IL-1A. (B) Expression profiles of 6 cytokine genes were assessed in the three drug-resistant cell lines by qRT-PCR analysis. Relative ratios of gene expression were calculated by comparison with IL-1A. (C) IL-8 and IL-18 gene expression in the six MPM cell lines is shown. Relative ratios of gene expression in the MPM cell lines were calculated by comparison with MET-5A cells.

96-well cell invasion assay (Cell Biolabs, Inc., San Diego, CA, USA). Gently, MESO1 cell suspension ( $2 \times 10^{5}$ cells) with or without either miR-379 or miR-411 mimic, or si-IL-18 in serum-free medium was added to the membrane chamber and incubated for $24 \mathrm{~h}$. The subsequent procedures were performed according to the manufacturer's protocol.

\section{Results}

Effect of PEM and SAHA treatment on the growth of MPM cells. We examined the antitumor activities of PEM and SAHA against six MPM cell lines. The $\mathrm{IC}_{50}$ values of PEM and SAHA against this panel of cell lines were determined by an MTS assay (Table I). One micromole is much lower than the mean peak plasma concentration of PEM achievable in patients, indicating a surprisingly high in vitro sensitivity of MPM cells to this agent (25). Based on relative sensitivity to PEM, these cell lines were classified as either sensitive $\left(\mathrm{IC}_{50}\right.$ of $\left.\leq 1 \mu \mathrm{M}\right)$ or resistant $\left(\mathrm{IC}_{50}\right.$ of $\left.>1 \mu \mathrm{M}\right)$. The $\mathrm{H} 28$ and $211 \mathrm{H}$ cell lines exhibited $\mathrm{IC}_{50}$ values of $0.1 \mu \mathrm{M}$ (highly sensitive). The H2052 cell line had an $\mathrm{IC}_{50}$ of 0.1 to $1 \mu \mathrm{M}$ (intermediate-sensitive). The PEM-resistant group included H2452, MESO1 and MESO4 cells. In relation to clinical trial data, PEM was shown to be effective in MPM patients with an epithelial histological type. However, on the basis of this in vitro study, PEM displayed antitumor effects against MPM independent of histological type. The antitumor activities of SAHA were also examined against the same panel of MPM cell lines (Table I). According to the highest concentration of the drug $\left(\mathrm{C}_{\max }\right)$ in patients treated with SAHA $(2-5 \mu \mathrm{M})$, these cell lines were classified as sensitive $\left(\mathrm{IC}_{50}\right.$ of $\left.\leq 5 \mu \mathrm{M}\right)$ or resistant $\left(\mathrm{IC}_{50}\right.$ of $\left.>5 \mu \mathrm{M}\right)$. Only the $211 \mathrm{H}$ cell line was deemed sensitive to SAHA (26). Based on the relative sensitivities against the two drugs, $211 \mathrm{H}$ cells were recognized as being commonly drug sensitive. The H28 and $\mathrm{H} 2052$ cell lines were classified as intermediate-sensitive cells. Finally, H2452, MESO1 and MESO4 cells were deemed as being commonly resistant to both agents.

IL-18 as a marker of resistance to SAHA. We next performed gene expression profiling of the same set of six MPM cell lines by GeneChip analysis. We used the MTS results for PEM and SAHA to develop a molecular model of drug sensitivity. Gene expression profiles were compared between the drug sensitive $211 \mathrm{H}$ cells and the five remaining cell lines displaying either intermediate or complete resistance to both agents. Sixteen genes were identified as being significantly correlated with the drug sensitivity (fold change $>1.5 ; \mathrm{P}<0.05$; Fig. 1A). Next, pathway analysis was performed using 16 genes to provide a viewpoint of the biological function of the identified differentially expressed genes, as previously described (27). Sixteen genes, associated with chemosensitivity, were identified based on the biological functions of the altered/associated genes (Fig. 1B). Pathway analysis identified IL-18 as an important gene associated with drug sensitivity 
A $211 \mathrm{H}$ H2052 H28 H2452 MESO1 MESO4

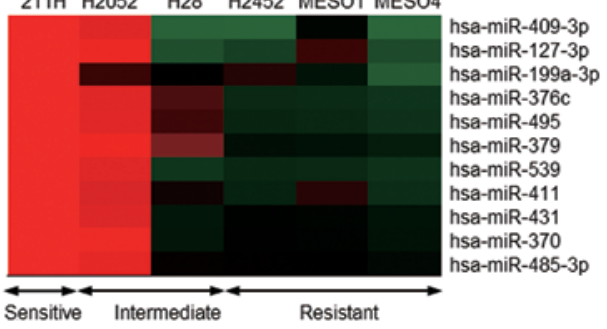

C

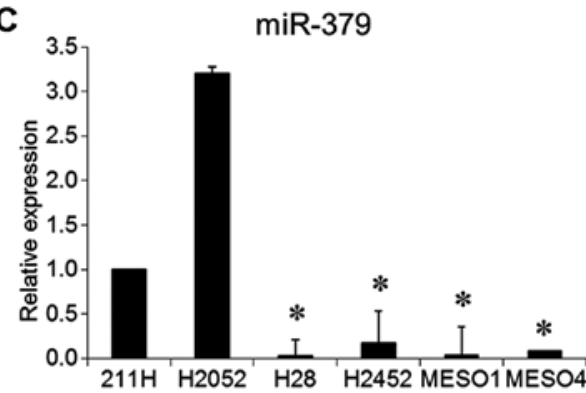

D

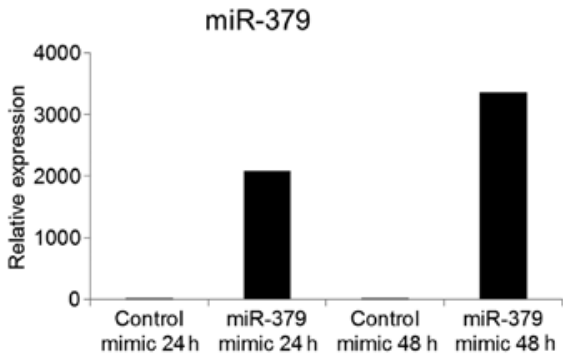

B Position 128-134 of IL18 3' UTR 5' ... UGgugaAaCCUCAUCUCUACUAA... 111111

hsa-miR-411

3.

GCAUGCGAUAUGCCAGAUGAU
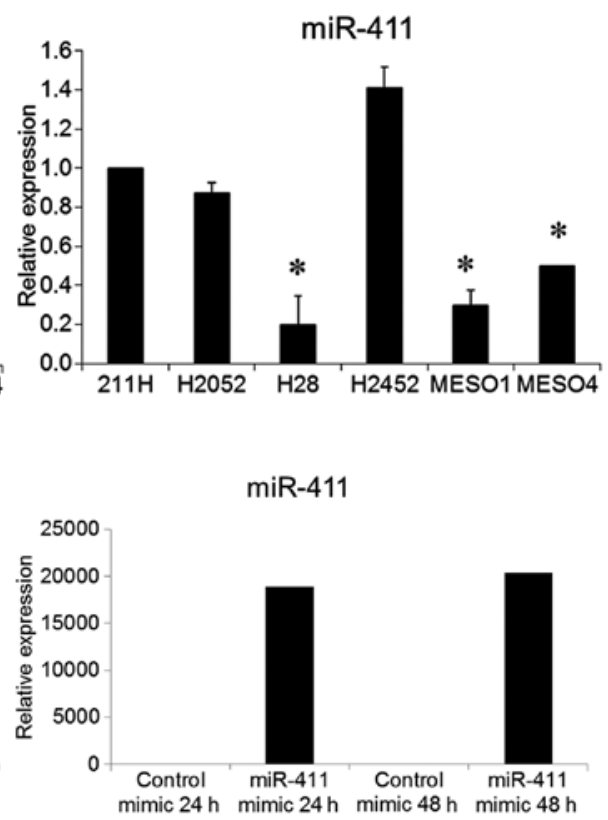

E

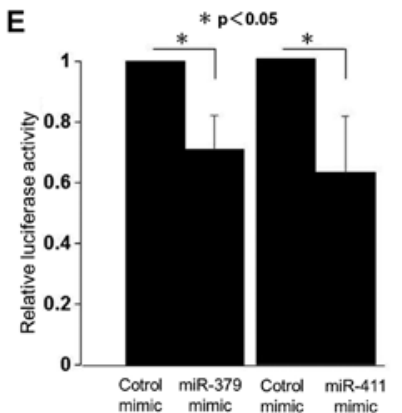

$\mathbf{F}$

Figure 3. miR-379 and miR-411 directly target IL-18 expression. (A) miRNAs that were differentially expressed between the drug-sensitive and -resistant MPM cell lines. Clustering analysis revealed that 11 miRNAs were differentially expressed between the drug-sensitive $211 \mathrm{H}$ cell line and the drug-resistant cell lines. (B) Predicted duplex formations by human IL-18 3' UTR and miR-411, as determined by TargetScan. (C) miR-379 and miR-411 expression levels were significantly lower in the drug-resistant and intermediate-sensitive cell lines than levels in the sensitive $211 \mathrm{H}$ cell line, as evaluated by qRT-PCR analysis. ${ }^{*} \mathrm{P}<0.05$, compared with the $211 \mathrm{H}$ cells (paired t-test). (D) Efficiency of transfection with miR-379 and miR-411 mimics. $\mathrm{H} 28$ cells were transfected with $50 \mathrm{~nm}$ of miR-379 mimics or miR-411 mimics for 24 to $48 \mathrm{~h}$. qRT-PCR analysis revealed that the mature forms of miR-379 and miR-411 were markedly induced following introduction of miR-379 and miR-411 mimics into the H28 cells. (E) miR-379 and miR-411 suppressed the luciferase activity of the pGL3-IL-18 3' UTR reporter. H28 cells were co-transfected with the pGL3-IL-18 3' UTR reporter and miR-379 mimics, and dual luciferase assays were carried out $48 \mathrm{~h}$ after transfection. H28 cells were co-transfected with the pGL3-IL-18 3' UTR reporter and miR-411 mimics, and dual luciferase assays were carried out $24 \mathrm{~h}$ after transfection. Data are expressed as the mean values $\pm \mathrm{SD}$ from 3 independent experiments. " $\mathrm{P}<0.05$ compared with the control cells (paired t-test). (F) Following transfection of $\mathrm{H} 28$ cells either with miR-379 mimics or miR-411 mimics for 24 to $48 \mathrm{~h}$, the expression levels of IL-18 mRNA were significantly reduced according to qRT-PCR analysis.

of MPM cells (Fig. 1B). IL-18 gene expression levels in the resistant cell lines and those of intermediate sensitivity were significantly higher than that found in the drug-sensitive $211 \mathrm{H}$ cells (Fig. 1A). Based on the results of DNA microarray and pathway analysis, we chose IL-18 for further analysis of drug resistance in MPM cells.
Cytokine gene expression profiles in MPM cells. To investigate the role of IL-18 in promoting drug resistance in the context of MPM treatment, we analyzed the expression of IL-18 and the other 9 cytokine genes in the same panel of cell lines. Among the cytokines evaluated, IL-2, IL-4, IL-10 and IL-27 mRNA expression was undetectable in practically all MPM cell lines tested. 


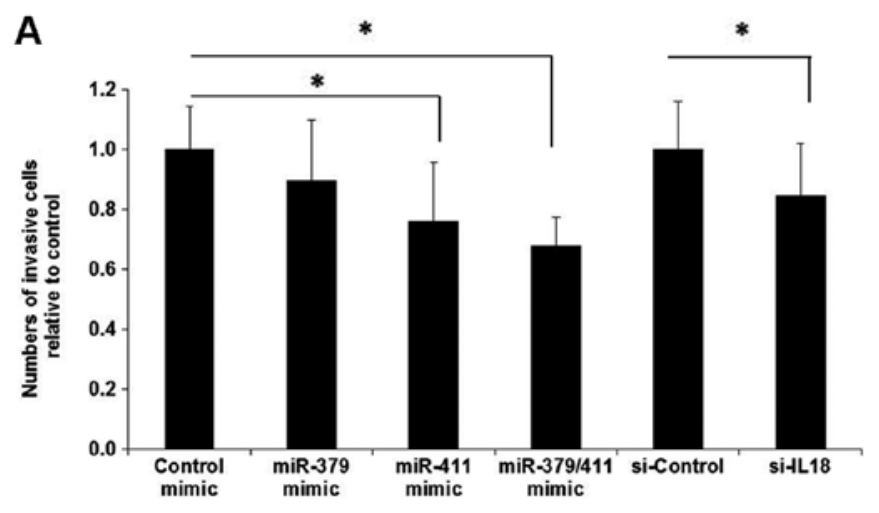

B
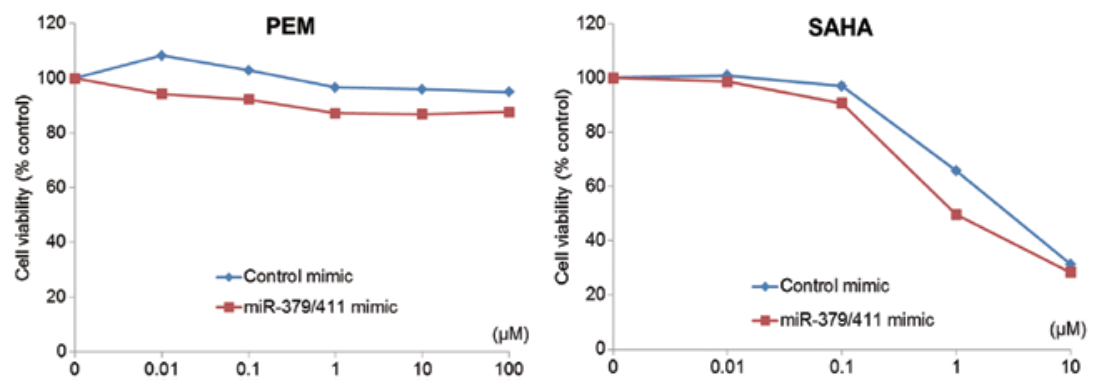

Figure 4. Effect of miR-379 and miR-411 expression on the invasive capacity and resistance to PEM and SAHA in MPM cells. (A) In vitro invasion assay. Invasive capacity of MESO1 cells after transfection with miR-411 mimics, miR-379/411 mimic combination and si-IL-18 was significantly decreased compared with that observed in the parental MESO1 cells. ${ }^{*} \mathrm{P}<0.05$ compared with the control cells (paired t-test). (B) Drug-resistant MESO1 cells with high IL-18 expression treated with miR-379/miR-411 mimic combination for $24 \mathrm{~h}$ were further incubated with various concentrations of PEM and SAHA for $72 \mathrm{~h}$. Each result is expressed as cell viability in the treated samples compared with the untreated sample (100\%) for PEM and SAHA therapy. Data are the mean values \pm SD from 3 independent experiments.

Therefore, we evaluated the correlation between the expression of the remaining 6 cytokine genes and drug sensitivity in the MPM cells (Fig. 2A and B). In three drug-sensitive and intermediate MPM cell lines including 211H, H2052 and H28 cells, IL-8 gene expression levels were highest among the 6 cytokine genes in the three PEM-sensitive cell lines (Fig. 2A and C). In contrast, IL-18 was the most upregulated compared to the other 5 cytokine genes in the three drug-resistant cell lines (Fig. 2B and C). Therefore, IL-18 was investigated further as a candidate marker of drug sensitivity.

Regulation of IL-18 expression via miR-379 and miR-411. We next examined miRNA expression profiles in the MPM cell lines to clarify the mechanism of IL-18 induction in drug-resistant MPM cell lines. The expression levels of 11 miRNAs in the three drug-resistant cell lines were increased significantly more than that observed in the $211 \mathrm{H}$ cells (fold change $>10.0$; Fig. $3 \mathrm{~A}$ ). We next proceeded to identify potential targets using the Targetscan and miRNA.org database, comprehensive resource of miRNA target predictions and expression profiles. We found that miR-379 and miR-411 belonged to the same cluster of miRNAs located on chromosome $14 \mathrm{q} 32$ that commonly target $I L-18$. Fig. 3B shows the regions within the $3^{\prime}$ UTR of the IL-18 gene that could serve as binding sites for miR-411 based on Targetscan predictions. We validated the downregulation of miR-379 expression in the three drug-resistant cell lines, as well as reduction in miR-411 expression in the two drug-resistant cell lines, using qRT-PCR analysis (Fig. 3C).
Next, we performed a luciferase reporter assay to verify that miR-379 and miR-411 directly target IL-18. Mature miR-379 and miR-411 in the H28 cells were significantly increased from 24 to $48 \mathrm{~h}$ after transfection of the relevant mimics (Fig. 3D). We found that co-transfection of either the miR-379 or miR-411 mimic with the IL-18 3' UTR reporter vector significantly decreased luciferase activity in the $\mathrm{H} 28$ cells as compared with the control (Fig. 3E). In addition, qRT-PCR analysis showed that treatment with either the miR-379 or miR-411 mimic induced downregulation of IL-18 mRNA expression in the $\mathrm{H} 28$ cells from 24 to $48 \mathrm{~h}$ (Fig. 3F). These data showed that IL-18 is a direct target of miR-379 and miR-411.

miR-379 and miR-411 inhibit the invasive activity in MPM cells and improve sensitivity to SAHA. We then examined the function of miR-379 and miR-411 in an invasion assay using MESO1 cells which express these miRNAs at a constitutively low level. Introduction of miR-411, as well as IL-18 silencing, significantly suppressed the invasive activities of these cells in vitro (Fig. 4A). Furthermore, the combination of both miR-379 and miR-411 mimics further inhibited the invasive capacity of these cells (Fig. 4A). Finally, we evaluated whether miR-379 and miR-411 elicited resistance to PEM and SAHA. Introduction of the combined miR-379/miR-411 mimics into MESO1 cells mediated a trend towards improved sensitivity to PEM (Fig. 4B). It is noteworthy that the same combination of miR-379/miR-411 mimics also appeared to increase sensitivity to SAHA (Fig. 4B). The $\mathrm{IC}_{50}$ values of 
miR-379/miR-411-transfected and parental MESO1 cells were 1.0 and $8.1 \mu \mathrm{M}$, respectively. Indeed, the $\mathrm{IC}_{50}$ value of the miR-379/miR-411-transfected MESO1 cells was below that observed in the drug-sensitive $211 \mathrm{H}$ cells $(3.3 \mu \mathrm{M})$. These findings suggest that miR-379 and miR-411 play a key role in the carcinogenesis of MPM cells by targeting IL-18 and contributing to the sensitivity of MPM cells to SAHA and PEM.

\section{Discussion}

We investigated whether specific genes and miRNAs could be useful as biomarkers of the drug response in MPM. In this study, IL-18 was identified as a drug-resistant gene by whole transcriptome and targeted cytokine gene expression profiling of MPM cell lines. IL-18 was originally discovered as an IFN- $\gamma$-inducing factor (12). IL-18 combines with IL-12 and induces IFN- $\gamma$ production by $\mathrm{T}$ and $\mathrm{NK}$ cells, mediates Th1 polarization and is involved in mediating defense to pathogens (13). IL-18 also combines with IL-2 and exhibits antitumor properties through the induction of an immune response (14). In addition, IL-18 alone can promote angiogenesis, metastasis, and escape from the immune response in the absence of Th1-like cytokines. In several human cancers, increased IL-18 serum levels accompany tumor progression and contribute to a poor prognosis (28). These reports indicate that IL-18 is an important cytokine in respect to the promotion of human cancer progression and metastasis.

In the present study, expression levels of miR-379 and miR-411, which are located on the same cluster, were significantly decreased in the drug-resistant MPM cell lines. A decreased level of miR-379 has been implicated in breast cancer $(29,30)$. Indeed, miR-379 has been shown to regulate cyclin B1 and TGF- $\beta$-induced IL-11 production in breast cancer cell lines $(29,30)$. Our results indicate that IL-18 is a direct target of miR-379 and miR-411. Transfection of MPM cells with miR-379 and miR-411 mimics resulted in decreased IL-18 expression, suppressed invasive capacity and contributed to resistance to SAHA and PEM. Our results provide the first integrated evidence for a significant role of miR-379 and miR-411 in the carcinogenesis of MPM, while also defining these as potentially novel drug targets.

Combination chemotherapy of PEM with CDDP is currently the best treatment available for MPM patients; however, its effectiveness is limited (5). New therapeutic agents are required for MPM patients. HDAC inhibitors are emerging as candidate therapeutic agents in this arena based on the results of in vitro and in vivo studies $(6,7)$. In our study, high levels of IL-18 were found in PEM- and SAHA-resistant MPM cell lines. IL-18 silencing by transfection of miR-379/miR-411 mimics mediated a trend towards improved sensitivity to PEM and SAHA in MPM cells that have constitutively high levels of IL-18. Elevated levels of IL-18 have been shown in doxorubicin-resistant breast cancer cell lines (31). A recent report revealed that depletion or neutralization of IL-18 decreased NK cell-controlled tumor metastasis in a PD-1 dependent manner, and suggested the possibility of novel clinical development of anti-PD1 antibodies in human malignancies that produce IL-18 (32). Therefore, IL-18 targeted therapy may be a novel potential therapeutic strategy in MPM cells with high IL-18. Furthermore, miR-379 and miR-411 could be used as a therapeutic intervention to regulate the expression of IL-18 and further control tumor invasion of MPM cells.

In conclusion, we provide insight into the possible contribution of the miR-379/411 cluster and IL-18 interaction in MPM cells. Our data suggest that IL-18 is a key determinant of sensitivity to PEM and SAHA in MPM cells. The miR-379/411 cluster promoted invasion and induced resistance to PEM and SAHA by directly targeting IL-18 in MPM cells. The miR-379/411 cluster may represent a new therapeutic target for advanced MPM patients, depending on the status of IL-18 expression. Further studies should be undertaken to clarify the mechanism underlying the association between the miR-379/411 cluster and drug resistance in MPM.

\section{Acknowledgements}

We would like to thank Ms. Shoko Tanaka for assistance with the cytokine gene expression analysis. We would like to thank Mrs. Haruka Isobe of MediBic for assistance with the Pathway analysis. This study was supported in part by a Grant-in-Aid from the Ministry of Education, Culture, Sports, Science and Technology of Japan (grant no. 24591179 to M.S.; grant no. 25461172 to A.G.), the Clinical Rebiopsy Bank Project for Comprehensive Cancer Therapy Development (to M.S and A.G.) and the Smoking Research Foundation (to A.G.).

\section{References}

1. Kaufman AJ and Pass HI: Current concepts in malignant pleural mesothelioma. Expert Rev Anticancer Ther 8: 293-303, 2008.

2. Hansen J, de Klerk NH, Musk AW and Hobbs MS: Environmental exposure to crocidolite and mesothelioma: exposure-response relationships. Am J Respir Crit Care Med 157: 69-75, 1998.

3. Robinson BWS and Lake RA: Advances in malignant mesothelioma. N Engl J Med 353: 1591-1603, 2005.

4. Sugarbaker DJ, Flores RM, Jaklitsch MT, et al: Resection margins, extrapleural nodal status, and cell type determine postoperative long-term survival in trimodality therapy of malignant pleural mesothelioma: results in 183 patients. J Thorac Cardiovasc Surg 117: 54-63, 1999.

5. Vogelzang NJ, Rusthoven JJ, Symanowski J, et al: Phase III study of PEM in combination with cisplatin versus cisplatin alone in patients with malignant pleural mesothelioma. J Clin Oncol 21: 2636-2644, 2003.

6. Hurwitz JL, Stasik I, Kerr EM, et al: Vorinostat/SAHAinduced apoptosis in malignant mesothelioma is FLIP/caspase 8-dependent and HR23B-independent. Eur J Cancer 48: 1096-1107, 2012.

7. Vandermeers F, Hubert P, Delvenne P, et al: Valproate, in combination with pemetrexed and cisplatin, provides additional efficacy to the treatment of malignant mesothelioma. Clin Cancer Res 15: 2818-2828, 2009.

8. Ramalingam SS, Parise RA, Ramanathan RK, et al: Phase I and pharmacokinetic study of vorinostat, a histone deacetylase inhibitor, in combination with carboplatin and paclitaxel for advanced solid malignancies. Clin Cancer Res 13: 3605-3610, 2007.

9. Zitvogel L, Tesniere A and Kroemer G: Cancer despite immunosurveillance: immunoselection and immunosubversion. Nat Rev Immunol 6: 715-727, 2006.

10. Zou W: Immunosuppressive networks in the tumour environment and their therapeutic relevance. Nat Rev Cancer 5: 263-274, 2005.

11. Seike M, Yanaihara N, Bowman ED, et al: Use of a cytokine gene expression signature in lung adenocarcinoma and the surrounding tissue as a prognostic classifier. J Natl Cancer Inst 99: 1257-1269, 2007.

12. Okamura H, Tsutsi H, Komatsu T, et al: Cloning of a new cytokine that induces IFN-gamma production by $\mathrm{T}$ cells. Nature 378: 88-91, 1995. 
13. Coughlin CM, Salhany KE, Wysocka M, et al: Interleukin-12 and interleukin-18 synergistically induce murine tumor regression which involves inhibition of angiogenesis. J Clin Invest 101: 1441-1452, 1998

14. Son YI, Dallal RM, Mailliard RB, Egawa S, Jonak ZL and Lotze MT: Interleukin-18 (IL-18) synergizes with IL-2 to enhance cytotoxicity, interferon-gamma production, and expansion of natural killer cells. Cancer Res 61: 884-888, 2001.

15. Park S, Cheon S and Cho D: The dual effects of interleukin-18 in tumor progression. Cell Mol Immunol 4: 329-335, 2007

16. Johnson SM, Grosshans H, Shingara J, et al: Ras is regulated by the let-7 microRNA family. Cell 120: 635-647, 2005.

17. Lu J, Getz G, Miska EA, Alvarez-Saavedra E, et al: MicroRNA expression profiles classify human cancers. Nature 435: 834-838, 2005.

18. Yanaihara N, Caplen N, Bowman E, et al: Unique microRNA molecular profiles in lung cancer diagnosis and prognosis. Cancer Cell 9: 189-198, 2006.

19. Seike M, Goto A, Okano T, et al: MiR-21 is an EGFR-regulated anti-apoptonic factor in lung cancer in never-smokers. Proc Natl Acad Sci USA 106: 12085-12090, 2009.

20. Pass HI, Goparaju C, Ivanov S, et al: hsa-miR-29c* is linked to the prognosis of malignant pleural mesothelioma. Cancer Res 70 1916-1924, 2010

21. Cioce M, Ganci F, Canu V, et al: Protumorigenic effects of miR-145 loss in malignant pleural mesothelioma. Oncogene: Nov 18, 2013 (Epub ahead of print). doi: 10.1038/onc.2013.476.

22. Shimokawa T, Seike M, Soeno C, et al: Enzastaurin has antitumour effects in lung cancers with overexpressed JAK pathway molecules. Br J Cancer 106: 867-875, 2012.

23. Gemma A, Li C, Sugiyama Y, et al: Anticancer drug clustering in lung cancer based on gene expression profiles and sensitivity database. BMC Cancer 6: 174, 2006.
24. Kitamura K, Seike M, Okano T, et al: MiR-134/487b/655 cluster regulates TGF- $\beta$-induced epithelial-mesenchymal transition and drug resistance to gefitinib by targeting MAGI2 in lung adenocarcinoma cells. Mol Cancer Ther 13: 444-453, 2014.

25. Thodtmann R, Depenbrock H, Dumez H, et al: Clinical and pharmacokinetic phase I study of multitargeted antifolate (LY231514) in combination with cisplatin. J Clin Oncol 17: 3009-3016, 1999.

26. Fakih MG, Pendyala L, Fetterly G, et al: A phase I, pharmacokinetic and pharmacodynamic study on vorinostat in combination with 5-fluorouracil, leucovorin, and oxaliplatin in patients with refractory colorectal cancer. Clin Cancer Res 15: 3189-3195, 2009.

27. Miyanaga A, Gemma A, Noro R, et al: Antitumor activity of histone deacetylase inhibitors in non-small cell lung cancer cells: development of a molecular predictive model. Mol Cancer Ther 7: 1923-1930, 2008

28. Dinarello CA: The paradox of pro-inflammatory cytokines in cancer. Cancer Metastasis Rev 25: 307-313, 2006.

29. Pollari S, Leivonen SK, Perälä M, Fey V, Käkönen SM and Kallioniemi O: Identification of microRNAs inhibiting TGF- $\beta$ induced IL-11 production in bone metastatic breast cancer cells. PLoS One 7: e37361, 2012.

30. Khan S, Brougham CL, Ryan J, et al: miR-379 regulates cyclin B1 expression and is decreased in breast cancer. PLoS One 8: e68753, 2013.

31. Yao L, Zhang Y, Chen K, et al: Discovery of IL-18 as a novel secreted protein contributing to doxorubicin resistance by comparative secretome analysis of MCF-7 and MCF-7/Dox. PLoS One 6: e24684, 2011.

32. Terme M, Ullrich E, Aymeric L, et al: IL-18 induces PD-1dependent immunosuppression in cancer. Cancer Res 71: 5393-5399, 2011. 